\title{
Gastric and Esophageal Cancers: Guidelines Updates
}

\author{
Presented by Crystal S. Denlinger, MD; Kristina A. Matkowskyj, MD, PhD; and Mary F. Mulcahy, MD
}

\section{ABSTRACT}

For the treatment of gastric and esophageal cancers, several pivotal trials_especially those evaluating immune checkpoint inhibitors (ICls)_ have altered the treatment landscape and led to changes in the NCCN Guidelines. In addition to pembrolizumab and nivolumab, new treatment options include trastuzumab-deruxtecan (T-DXd), ramucirumab, and trifluridine/tipiracil. These agents convey varying degrees of benefit depending on treatment line, PD-L1 expression, HER2 expression, and tumor histology. Recently, ICls have been incorporated into the first-line treatment of HER2-negative advanced esophageal, gastroesophageal junction (GEJ), and gastric cancers, in addition to second-line treatment of advanced esophageal and GEJ cancer of squamous histology. T-DXd is another new second-line option for HER2-positive esophageal, GEJ, and gastric adenocarcinomas. ICls are now moving into the adjuvant setting as well, and a new recommendation is nivolumab use after preoperative chemoradiation and surgery in patients who have residual disease identified at the time of their R0 resections.

J Natl Compr Canc Netw 2021;19(5.5):639-643 doi: $10.6004 /$ jnccn.2021.5006

For the treatment of gastric and esophageal cancers, recent studies have demonstrated robust findings for new drugs that, as a consequence, are becoming FDAapproved and added to the NCCN Clinical Practice Guidelines in Oncology (NCCN Guidelines) for Esophageal and Esophagogastric Junction Cancers and for Gastric Cancer. These new drugs include immune checkpoint inhibitors (ICIs), the HER2-directed agent trastuzumab-deruxtecan (T-DXd), the monoclonal antibody ramucirumab, and the cytotoxic agent trifluridine/tipiracil. Trials supporting the use of these improved therapies, and their application in the clinic for certain subgroups, was presented by Crystal S. Denlinger, MD, Chief, Division of Gastrointestinal Medical Oncology, and CoDirector, Survivorship Program, and Associate Professor, Department of Hematology-Oncology, Fox Chase Cancer Center, and Mary F. Mulcahy, MD, Professor, Department of Hematology-Oncology, Northwestern University, and Director, Gastrointestinal Oncology Program, Robert H. Lurie Comprehensive Cancer Center of Northwestern University, at the NCCN 2021 Virtual Annual Conference.

Furthermore, the growing importance of biomarkers in these cancers was emphasized by Kristina A. Matkowskyj, MD, PhD, Associate Professor in the Department of Pathology and Laboratory Medicine and Director of the UW Carbone Cancer Center Translational Sciences BioCore. Because these malignancies are now being categorized according to HER2 status, microsatellite instability-high (MSI-H) or mismatch repair deficient (dMMR) status, and PD-L1 expression, biomarker testing is a necessary first step at diagnosis to facilitate treatment management across the therapy continuum. Tumor histology remains a critical factor in decision-making; biomarker testing for HER2 is performed only on adenocarcinomas, whereas drug selection for a given line of therapy for squamous cell carcinoma and adenocarcinoma may differ based on PD-L1 expression levels, she said.

These treatment advances have greatly expanded the treatment landscape. "Certainly, we now have options," Dr. Denlinger commented. "I remember the time when we had one line of therapy; now we are talking about multiple options in second and later lines, so we can tailor our regimens, not only to disease characteristics but also to patient characteristics."

\section{Considerations From a Pathologist}

With the advent of new treatments, biomarker testing of newly diagnosed tumors is pivotal. Dr. Matkowskyj noted that biomarker testing can be performed on formalinfixed, paraffin-embedded tissue, with samples stored for at least 10 years unless exhausted for testing or clinical trial enrollment. This makes it possible to return to the tissue to retest, "should new biomarkers arise," she said.

The most critical biomarker testing includes (1) HER2 by immunohistochemistry (IHC), with reflex testing to in situ hybridization (ISH) if the original result is reported as equivocal; (2) microsatellite instability testing by PCR or mismatch repair (MMR) expression by IHC; (3) PD-Ll expression by IHC; and (4) next-generation sequencing when the above sequential testing cannot be performed when tissue is limited or if the aforementioned tests have been performed and additional tissue remains. 
Dr. Matkowskyj emphasized the importance of PDL1 testing in the esophagogastric setting and advised listeners on some of the finer points. In this setting, PD-L1 expression is calculated and reported as combined positive score (CPS) based on the examination of $\geq 100$ tumor cells. CPS is the number of PD-L1 staining cells (ie, tumor cells, lymphocytes, macrophages) divided by the total number of viable tumor cells, multiplied by 100. A CPS $\geq 1$ is indicative of positive PD-L1 expression. In contrast, other cancer types use total positive score (TPS), which is a separate scoring system. It is important for clinicians to specify the primary tumor site when ordering PD-L1 testing so that pathologists can perform the analysis using the correct scoring system, she reminded listeners.

\section{First-Line Therapy for Advanced Disease: CheckMate 649}

"Based on early results showing the effect of immunotherapy, we've seen a series of first-line studies that were quickly up and running," Dr. Mulcahy said. For the first-line treatment of advanced gastric cancer, gastroesophageal junction (GEJ) cancer, or esophageal adenocarcinoma, results of the phase III CheckMate 649 trial were important. ${ }^{1}$ This trial enrolled 1,581 patients with HER2-negative advanced or metastatic gastric, gastroesophageal, or esophageal adenocarcinoma to chemotherapy with fluoropyrimidine and oxaliplatin (FU/leucovorin/oxaliplatin [FOLFOX] or capecitabine/oxaliplatin [XELOX] \pm nivolumab. The dual primary endpoints were overall survival (OS) and progression-free survival (PFS) for subjects with PD-L1 CPS $\geq 5$. Secondary endpoints evaluated OS and PFS for CPS $\geq 1, \mathrm{CPS} \geq 10$, all randomized, and response rate. Seventy percent of enrolled subjects had gastric cancer and $60 \%$ of enrolled subjects had PD-L1 CPS $\geq 5$. Median OS was significantly increased with nivolumab + chemotherapy (14.4 vs 11.1 months with chemotherapy alone; hazard ratio [HR], $0.71 ; P<.0001$ ); 12 -month survival was $57 \%$ vs $46 \%$, respectively, and this benefit was sustained over time. Response rates were also higher and more durable with the combination.

"More than $80 \%$ of patients had CPS $\geq 1$, so this was a population somewhat enriched for PD-L1 expression," Dr. Mulcahy noted. "We see that for CPS $\geq 1$, benefit was still maintained (HR, 0.77; $P=.00001$ ), but we interpret this with caution, as within this group $60 \%$ had CPS $\geq 5$, meaning this population was heavily weighted [toward enrichment]. As you broaden the patient population, the HR gets closer to $1 . "$

In this patient population, nivolumab is the first PD-1 inhibitor to demonstrate superior OS and PFS in combination with chemotherapy versus chemotherapy alone, and represents a new potential standard first-line treatment for the subgroup with CPS $\geq 5$, Dr. Mulcahy indicated.

Dr. Denlinger noted that these encouraging findings stand in contrast to the dichotomous results from 2 other studies evaluating ICIs with chemotherapy: KEYNOTE$062,{ }^{2}$ which evaluated pembrolizumab + cisplatin/FU or capecitabine, and ATTRACTION-4, ${ }^{3,4}$ which evaluated nivolumab + oxaliplatin and S1 or capecitabine. The negative results of these studies may be attributed to the smaller size of the studies, the lack of PD-L1 enrichment, and the fact that more patients received subsequent therapies compared with CheckMate 649, she suggested (Table 1).

"I think CheckMate 649 informs our new treatment decisions, but we do have to take into account that not every patient will derive as much benefit as patients with high CPS scores," Dr. Denlinger said.

\section{First-Line Treatment for Esophageal Cancer: KEYNOTE-590}

In treatment-nave advanced esophageal cancer, ICIs also have a role, as demonstrated by the results observed with the addition of pembrolizumab to FU + cisplatin in KEYNOTE-590. ${ }^{5}$ This trial included only patients with esophageal cancer of either adenocarcinoma or squamous cell carcinoma histology, and thus is quite informative for the esophageal population, Dr. Mulcahy said. Patients were randomized to chemotherapy + pembrolizumab or chemotherapy alone, and were continued on pembrolizumab or placebo after 6 cycles of chemotherapy. The dual primary endpoints were OS and PFS. Results demonstrated improved outcomes with pembrolizumab + chemotherapy compared with chemotherapy alone, including a median OS of 12.4 versus 9.8 months (HR, 0.73; $P<.0001$ ), respectively, and, among patients with CPS $\geq 10$, median OS was 13.5 versus 9.4 months (HR, 0.62; $P<.0001$ ), respectively. Furthermore, for the squamous cell subset, median OS was 12.6 versus 9.8 months (HR, $0.72 ; P=.0006$ ) and for those with CPS $\geq 10$, median OS was 13.9 versus 8.8 months, respectively (HR, 0.57; $P<.0001$ ) (Table 2).

However, she continued, there are caveats with KEYNOTE-590: $70 \%$ of patients had squamous histology, which is not particularly common in the US population; the chemotherapy was 5-FU + cisplatin, which has largely been replaced by oxaliplatin-based therapy in the United States; and although patients with any PD-L1 expression could enroll, $50 \%$ did have high expression (ie, CPS $\geq 10$ ).

Caveats aside, Dr. Mulcahy concluded, "This is definitely a study demonstrating a benefit for ICIs in this particular population.... In all patients, including those with CPS $<10$, you still see a sustained benefit, though it dampened some."

\section{Recent Advances in HER2-Positive Gastric/GEJ Cancer}

Moving on to HER2-positive cancer, Dr. Matkowskyj emphasized the importance of identifying HER2 positivity on 


\section{Table 1. Comparison of Phase III Trials in Gastric/GEJ Cancer}

\begin{tabular}{|c|c|c|c|}
\hline & KEYNOTE-062 ${ }^{2}$ & ATTRACTION-4 ${ }^{3,4,19}$ & CheckMate $649^{1,19}$ \\
\hline Sample size & 763 & 724 & 1,581 \\
\hline Geographic location & $\begin{array}{l}58 \% \text { European/ } \\
\text { North American }\end{array}$ & $\begin{array}{l}100 \% \text { Japan/ } \\
\text { Korea/Taiwan }\end{array}$ & $\begin{array}{l}\text { 75\% non-Asian } \\
25 \% \text { Asian }\end{array}$ \\
\hline Tumor location breakdown & $\begin{array}{l}69.1 \% \text { gastric } \\
30.9 \% \text { GEJ }\end{array}$ & $\sim 89 \%$ gastric & $\begin{array}{c}70 \% \text { gastric } \\
18 \% \text { GEJ } \\
13 \% \text { esophageal }\end{array}$ \\
\hline Chemo backbone & Cisplatin/5-FU or cape & Oxal/S1 or cape & Oxal/5-FU or cape \\
\hline Enrolled & PD-L1-positive only & All-comers & All-comers \\
\hline MSI-high & $6.6 \%$ & - & $4 \%$ \\
\hline Primary study population & $\mathrm{CPS} \geq 1$ & All-comers & $\mathrm{CPS} \geq 5$ \\
\hline PD-L1 antibody & 22C3 pharmDx (Dako) & 28-8 pharmDx (Dako) & 28-8 pharmDx (Dako) \\
\hline PD-L1 scoring methods & CPS & TPS & CPS \\
\hline$\%$ high PD-L1 & $\begin{array}{c}36.8 \% \text { CPS } \geq 10 \\
100 \% \text { CPS } \geq 1\end{array}$ & $\begin{array}{l}16 \% \text { tumor PD-L1-positive } \\
\quad \text { (tumor cell } \geq 1 \% \text { ) }\end{array}$ & $\begin{array}{l}60 \% \text { CPS } \geq 5 \\
82 \% \text { CPS } \geq 1\end{array}$ \\
\hline Subsequent therapies & $\sim 50 \%$ & $\sim 66 \%$ & $\sim 39 \%$ \\
\hline$\%$ received subsequent $\mathrm{IO}$ in trial arms & $14 \%$ control/4\% experimental & $27 \%$ control $/ 12 \%$ experimental & $8 \%$ control $/ 2 \%$ experimental \\
\hline
\end{tabular}

Abbreviations: cape, capecitabine; chemo, chemotherapy; CPS, combined positive score; GEJ, gastroesophageal junction; IO, immunotherapy; MSI, microsatellite instability; nivo, nivolumab; oxal, oxaliplatin; pembro, pembrolizumab; TPS, total positive score.

biopsy samples in order to select patients for new treatments. As she explained, a HER2-positive IHC result (score $3+$ ) is defined by a cluster of $\geq 5$ cancer cells with a strong complete, basolateral, or lateral membranous reactivity, irrespective of percentage of cancer cells that are positive. The sample is scored $2+$, or equivocal, when the same cluster of $\geq 5$ cancer cells show weak to moderate reactivity. When reactivity is faint, barely perceptible, or absent, the tumor is HER2-negative. The NCCN Guidelines recommend that IHC be performed first, followed by ISH in equivocal $(2+)$ cases to determine whether HER2 is amplified, she said.

When patients with HER2-positive gastric cancer experience disease progression after a trastuzumabcontaining regimen, new treatment options now exist that come on the heels of several negative phase II studies: the TyTAN trial of paclitaxel + lapatinib, ${ }^{6}$ the
GATSBY trial of trastuzumab emtansine (T-DM1) versus a taxane, ${ }^{7}$ and the T-ACT trial of paclitaxel + trastuzumab. ${ }^{8}$ "We have previously been disappointed in the available options in HER2-positive disease. Unlike in breast cancer, where HER2-directed therapy is effective across treatment lines, until recently we've had no data to continue antiHER2 therapy after progression on trastuzumab," Dr. Denlinger said. "But a new drug has been approved for the second or later line for HER2-positive gastric cancer."

T-DXd is an antibody-drug conjugate that links a humanized anti-HER2 antibody to a cytotoxic topoisomerase 1 inhibitor, optimizing delivery of the cytotoxic agent to the tumor cell. The drug also conveys a bystander effect, essentially killing neighboring cancer cells. T-DXd was recently approved for use in refractory HER2-positive gastric cancer based on the randomized phase II

\begin{tabular}{|c|c|c|c|c|c|c|c|c|c|c|c|c|}
\hline & \multicolumn{3}{|c|}{$\operatorname{ESCC}(74 \%)$} & \multicolumn{3}{|c|}{ ESCC CPS $\geq 10$} & \multicolumn{3}{|c|}{ PD-L1CPS $\geq 10(51 \%)$} & \multicolumn{3}{|c|}{ All Patients } \\
\hline & Pembro & Placebo & $H^{a}$ & Pembro & Placebo & $H^{a}$ & Pembro & Placebo & $\mathrm{HR}^{\mathrm{a}}$ & Pembro & Placebo & $H R^{a}$ \\
\hline OS & $12.6 \mathrm{mo}$ & $9.8 \mathrm{mo}$ & 0.72 & $13.9 \mathrm{mo}$ & $8.8 \mathrm{mo}$ & 0.57 & $13.5 \mathrm{mo}$ & $9.4 \mathrm{mo}$ & 0.62 & $12.4 \mathrm{mo}$ & $9.8 \mathrm{mo}$ & 0.73 \\
\hline 24-mo OS & $29 \%$ & $17 \%$ & & $31 \%$ & $15 \%$ & & $31 \%$ & $15 \%$ & & $28 \%$ & $16 \%$ & \\
\hline PFS & $6.3 \mathrm{mo}$ & $5.8 \mathrm{mo}$ & 0.65 & & & & $7.5 \mathrm{mo}$ & $5.5 \mathrm{mo}$ & 0.51 & $6.3 \mathrm{mo}$ & $5.8 \mathrm{mo}$ & 0.65 \\
\hline $18 \mathrm{mo}$ PFS & $17 \%$ & $6 \%$ & & & & & $21 \%$ & $5 \%$ & & $16 \%$ & $6 \%$ & \\
\hline ORR & & & & & & & & & & $45 \%$ & $29.3 \%$ & \\
\hline Duration & & & & & & & & & & $8.3 \mathrm{mo}$ & $6.0 \mathrm{mo}$ & \\
\hline
\end{tabular}

Abbreviations: CPS, combined positive score; ESCC, esophageal squamous cell carcinoma; HR, hazard ratio; ORR, overall response rate; OS, overall survival; pembro, pembrolizumab; PFS, progression-free survival.

${ }^{\mathrm{a}} P$ values were statistically significant. 
DESTINY-Gastric01 study in which response rate, the primary endpoint, was significantly improved with T-DXd$51 \%$ vs $14 \%$ with physician's choice of chemotherapy (primarily irinotecan). ${ }^{9}$ Median OS increased to 12.5 months from 8.4 months with chemotherapy (HR, 0.59; $P=.01$ ), and PFS was 5.6 versus 3.5 months (HR, 0.47).

"Those of us who see a lot of patients with gastric cancer know they don't typically get much response to second-line therapy, so $51 \%$ is a clinically meaningful number," Dr. Denlinger noted. But T-DXd does have toxicity. Importantly, clinicians should monitor for interstitial lung disease and pneumonitis, which was observed in $10 \%$ of the study population, and initiate corticosteroids promptly, she emphasized.

Another fairly new second-line agent is ramucirumab, which in combination with paclitaxel was established as a standard in gastric and GEJ tumors in the RAINFALL trial. ${ }^{10}$ The more recent RAMIRIS study suggested that FOLFIRI (5-FU/leucovorin/irinotecan) may be an alternative backbone for patients who are not candidates for a taxane or whose disease progresses shortly after a taxane-containing regimen. ${ }^{11}$ In RAMIRIS, response rates, PFS, and OS were similar between FOLFIRI/ramucirumab and paclitaxel/ramucirumab, although PFS favored FOLFIRI/ramucirumab in patients who were docetaxel-pretreated in earlier lines of therapy. Although both backbones can be used, current evidence still favors paclitaxel and ramucirumab after progression on a trastuzumab-containing regimen, she said.

\section{$\mathrm{ICl}$ as Second- or Third-Line Therapy}

There are patient subpopulations in whom an ICI may be an option in the second line, Dr. Denlinger added. KEYNOTE-061, which evaluated pembrolizumab versus paclitaxel in patients with CPS $\geq 1$, was a negative study, but did show some benefit in patients with CPS $\geq 10$. $^{12}$ Pembrolizumab also showed some benefit as third-line therapy in the phase II KEYNOTE-059 trial, especially among patients with PD-L1-positive disease (defined at CPS $\geq 1$ ), further supporting PD-L1 positivity as a biomarker for response and durability of benefit. ${ }^{13}$

A subset analysis of KEYNOTE-061 also showed encouraging outcomes in patients with MSI-H tumors, for whom median OS was not reached and was 8.1 months with paclitaxel. ${ }^{14}$ Similar benefits in MSI-H subsets have been seen across the KEYNOTE trials, and other studies have found pembrolizumab performs well in patients with high tumor mutational burdens (TMBs), according to Dr. Denlinger. ${ }^{14-16}$

According to Dr. Matkowskyj, in gastric and esophageal cancers, TMB-high is defined as the presence of $\geq 10$ mutations per megabase, but "knowing how each vendor calculates TMB and their scoring cutoff values is important."

\section{Other Third-Line Options in Gastric/GEJ Cancer}

Yet another relatively new option for patients with refractory disease who are ineligible for ICIs in the third line and beyond is trifluridine/tipiracil (TAS-102), an oral agent composed of a nucleoside analogue, trifluridine, and a thymidine phosphorylase inhibitor, tipiracil. In the phase III TAGS trial, trifluridine/tipiracil demonstrated improved outcomes compared with best supportive care in highly refractory disease. ${ }^{17}$ Median OS was 5.7 versus 3.6 months (HR, 0.69; $P=.00058$ ), although median PFS was essentially unchanged (but statistically significant) and response rates were low and not statistically different when compared with placebo.

\section{Immunotherapy in the Curative-Intent Setting}

CheckMate 577 provided evidence that ICIs can also significantly impact localized disease. ${ }^{18}$ This global phase III study enrolled 794 patients with stage II/III esophageal or GEJ cancer (adenocarcinoma or squamous cell carcinoma) who achieved R0 resections after neoadjuvant chemoradiation but had residual pathologic disease in the surgical specimen. Patients were unselected for PD-L1 status, and $70 \%$ had PD-L1 expression $<1 \%$. Patients were randomized to nivolumab or placebo for up to 1 year and followed for 2 years. For the primary endpoint, disease-free survival, there was clear benefit for nivolumab as adjuvant therapy (22.4 vs 11.0 months; HR, 0.69; $P=.0003$ ), with the benefit sustained over time. Nivolumab was favored in all subgroups.

"This is the most robust data we have seen showing any benefit for adjuvant therapy after resection in esophageal cancer,” Dr. Mulcahy observed. "These results represent the first advance in years for these patients, potentially establishing adjuvant nivolumab as a new standard of care."

\section{Recent Changes to the NCCN Guidelines}

As a result of the studies described, there have been important changes in the NCCN Guidelines, which Dr. Mulcahy outlined. "We are not only getting more agents, but also getting more robust data supporting their use," she said. In newly diagnosed patients, postoperative therapy with nivolumab after preoperative chemoradiation and surgery in patients who have achieved $\mathrm{R} 0$ resections but have residual disease is a category 1 recommendation.

For the first-line treatment of advanced disease, patients are categorized as HER2-positive or HER2negative. For HER2-negative esophageal and GEJ cancers, several preferred regimens have been added, including fluoropyrimidine/oxaliplatin/nivolumab in patients with CPS $\geq 5$ (adenocarcinoma) (category 1); fluoropyrimidine/cisplatin/pembrolizumab in those with CPS $\geq 10$ (category 1 ) (squamous cell carcinoma 
or adenocarcinoma); and fluoropyrimidine/oxaliplatin/pembrolizumab in those with CPS $\geq 10$ (squamous cell carcinoma or adenocarcinoma). "KEYNOTE 590 used fluoropyrimidine/cisplatin + pembrolizumab, which is why it has a category 1 recommendation, but we felt it's comparable to convert that to fluoropyrimidine with oxaliplatin and pembrolizumab, since most patients tolerate that better," Dr. Mulcahy said.

For advanced gastric cancer, a new preferred first-line regimen for HER2-negative patients is fluoropyrimidine/ oxaliplatin/nivolumab in patients with CPS $\geq 5$ (category 1). In the second-line treatment of esophageal and GEJ cancers, 2 category 1 recommendations have been added for patients with squamous cell carcinoma: (1) nivolumab, and (2) pembrolizumab in patients with CPS $\geq 10$.
For patients with HER2-positive disease and adenocarcinoma, T-DXd is another new option, both for esophageal/ GEJ cancer and gastric cancer, in the second line or later.

Disclosures: Dr. Denlinger has disclosed serving as a scientific advisor for BeiGene, Bristol Myers Squibb Company, Exelixis Inc., Merck \& Co., Inc., and Zymeworks Inc.; and receiving honoraria from Eli Lilly and Company and Taiho Pharmaceuticals Co., Ltd. Dr. Matkowskyj has disclosed receiving consulting fees from Elephas Bio and McDowell \& Morrissette Professional Association. Dr. Mulcahy has disclosed no relevant financial relationships.

Correspondence: Crystal S. Denlinger, MD, Fox Chase Cancer Center, 333 Cottman Avenue, Philadelphia, PA 19111.

Email: crystal.denlinger@fccc.edu;

Kristina A. Matkowskyj, MD, PhD, University of Wisconsin Carbone Cancer Center, 600 Highland Avenue, L5/183 CSC, Madison, WI 53792. Email: matkowskyj@wisc.edu; and

Mary F. Mulcahy, MD, Robert H. Lurie Comprehensive Cancer Center of Northwestern University, 676 North St. Clair Street, Suite 850, Chicago, IL 60611. Email: mmulcahy@nm.org

\section{References}

1. Moehler M, Shitara K, Garrido M, et al. Nivolumab plus chemotherapy versus chemotherapy as first-line treatment for advanced gastric cancer/ gastroesophageal junction cancer/esophageal adenocarcinoma: first results of the CheckMate 649 study [abstract]. Ann Oncol 2020;31(Suppl 4):S1191. Abstract LBA6_PR.

2. Shitara K, Van Cutsem E, Bang Y, et al. Efficacy and safety of pembrolizumab or pembrolizumab plus chemotherapy vs chemotherapy alone for patients with first-line, advanced gastric cancer: the KEYNOTE-062 phase 3 randomized clinical trial. JAMA Oncol 2020;6:1571-1580.

3. Boku N, Ryu MH, Kato K, et al. Safety and efficacy of nivolumab in combination with S-1/capecitabine plus oxaliplatin in patients with previously untreated, unresectable, advanced, or recurrent gastric/gastroesophageal junction cancer: interim results of a randomized, phase II trial (ATTRACTION-4). Ann Oncol 2019;30:250-258.

4. Boku N, Ryu MH, Oh D, et al. Nivolumab plus chemotherapy versus chemotherapy alone in patients with previously untreated advanced or recurrent gastric/gastroesophageal junction (G/GEJ) cancer: ATTRACTION-4 (ONO-4538-37) study [abstract]. Ann Oncol 2020;31(Suppl 4): Abstract LBA7 PR.

5. Kato K, Sun JM, Shah MA, et al. Pembrolizumab plus chemotherapy versus chemotherapy as first-line therapy in patients with advanced esophageal cancer: the phase 3 KEYNOTE-590 study. Ann Oncol 2020;31(Suppl 4):S1192.

6. Satoh $\mathrm{T}, \mathrm{Xu} \mathrm{RH}$, Chung $\mathrm{HC}$, et al. Lapatinib plus paclitaxel versus paclitaxel alone in the second-line treatment of HER2-amplified advanced gastric cancer in Asian populations: TyTAN-a randomized, phase III study. J Clin Oncol 2014;21:2039-2049.

7. Thuss-Patience PC, Shah MA, Ohtsu A, et al. Trastuzumab emtansine versus taxane use for previously treated HER2-positive locally advanced or metastatic gastric or gastro-oesophageal junction adenocarcinoma (GATSBY): an international randomised, open-label, adaptive, phase $2 / 3$ study. Lancet Oncol 2017;18:640-653.

8. Makiyama A, Sagara K, Kawada J, et al. A randomized phase II study of weekly paclitaxel \pm trastuzumab in patients with HER2-positive advanced gastric or gastro-esophageal junction cancer refractory to trastuzumab combined with fluoropyrimidine and platinum: WJOG7112G (T-ACT) [abstract]. J Clin Oncol 2018;36(Suppl):Abstract 4011.

9. Shitara K, Bang YJ, Iwasa S, et al. Trastuzumab deruxtecan in previously treated HER2-positive gastric cancer. N Engl J Med 2020;382:2419-2430.

10. Fuchs CS, Shitara K, Di Bartolomeo M, et al. Ramucirumab with cisplatin and fluoropyrimidine as first-line therapy in patients with metastatic gastric or junctional adenocarcinoma (RAINFALL): a double-blind, randomized, placebo-controlled, phase 3 trial. Lancet Oncol 2019;20:420-435

11. Lorenzen S, Thuss-Patience PC, Pauligk $C$, et al. FOLFIRI plus ramucirumab versus paclitaxel plus ramucirumab as second-line therapy for patients with advanced or metastatic gastroesophageal adenocarcinoma with or without prior docetaxel: results from the phase II RAMIRIS study of the AIO [abstract]. J Clin Oncol 2020;38(Suppl):Abstract 4514.

12. Shitara $\mathrm{K}$, Özgüroğlu $\mathrm{M}$, Bang $\mathrm{YJ}$, et al. Pembrolizumab versus paclitaxel for previously treated, advanced gastric or gastro-oesophageal junction cancer (KEYNOTE-061): a randomised, open-label, controlled, phase 3 trial. Lancet 2018;392:123-133.

13. Fuchs CS, Toshihiko D, Jang RW, et al. Safety and efficacy of pembrolizumab monotherapy in patients with previously treated advanced gastric and gastroesophageal junction cancer: phase 2 clinical KEYNOTE059 trial. JAMA Oncol 2018;4:e180013.

14. Chao J, Fuchs CS, Shitara K, et al. Pembrolizumab (pembro) in microsatellite instability-high (MSI-H) advanced gastric/gastroesophageal junction cancer G/GEJ) by line of therapy [abstract]. J Clin Oncol 2020;38(Suppl):Abstract 430.

15. Chao J, Fuchs CS, Shitara K, et al. Assessment of pembrolizumab therapy for the treatment of microsatellite instability-high gastric or gastroesophageal junction cancer among patients in the KEYNOTE-059, KEYNOTE-061, and KEYNOTE-062 clinical trials [published online April 1, 2021]. JAMA Oncol, doi: 10.1001/jamaoncol 2021;0275

16. Shitara K, Özgüroğlu $M$, Bang YJ, et al. The association of tissue tumor mutational burden (tTMB) using the Foundation Medicine genomic platform with efficacy of pembrolizumab versus paclitaxel in patients (pts) with gastric cancer (GC) from KEYNOTE-061 [abstract]. J Clin Oncol 2020;38(Suppl):Abstract 4537.

17. Shitara K, Doi T, Dvorkin M, et al. Trifluridine/tipiracil versus placebo in patients with heavily pretreated metastatic gastric cancer (TAGS): a randomised, double-blind, placebo-controlled, phase 3 trial. Lancet Oncol 2018;19:1437-1448.

18. Kelly, RJ, Ajani JA, Kuzdzal J, et al. Adjuvant nivolumab in resected esophageal or gastroesophageal junction cancer (EC/GEJC) following neoadjuvant chemoradiation therapy (CRT): first results of the CheckMate 577 study [abstract]. Ann Oncol 2020;31:s1193-1194. Abstract LBA9_PR.

19. Smyth EC, Cervantes E, Addition of nivolumab to chemotherapy in patients with advanced gastric cancer: a relevant step ahead, but still many questions to answer. ESMO Open 2020;5:e001107. 